\title{
Efforts to Improve the Follow-up of Patients With Abnormal Papanicolaou Test Results
}

\author{
Bruce Block, MD, and Rutb Ann Branbam
}

Background: We describe and evaluate efforts to improve the follow-up care of patients with abnormal Papanicolaou test results at an academic family practice center at Shadyside Hospital in Pittsburgh.

Methods: From 1994 to 1996, 1796 patients received Papanicolaou testing; 147 (8 percent) of the smears had abnormal findings-16 percent had atypia, 83 percent had dysplasia, and 1 percent had carcinoma in situ. Patients received follow-up care based on a formalized protocol using educational input, logistic aids, and automated prompting.

Results: Overall follow-up success rates and colposcopy completion rates increased dramatically. Whereas 36 percent of patients with abnormal findings on Papanicolaou smears had been overdue for follow-up in 1990, only 13 percent were overdue in 1996 after our interventions. Patients assigned to Papanicolaou testing for follow-up of abnormal findings failed to receive a test in 9 of 45 (20 percent) cases, but those assigned to colposcopy follow-up failed to receive a test in only 10 of 102 (10 percent) of cases. Appointment failure rates at colposcopy clinic dropped from 56 percent in 1993 to 12 percent in 1996. Colposcopic biopsy was far superior to Papanicolaou test for detecting precursors of cervical cancer at follow-up.

Conclusions: Educational programs, formalized approaches to care, transportation assistance, and reminder systems are not only practical but also can dramatically improve the outcome of cervical cancer screening programs. (J Am Board Fam Pract 1998;11:1-11.)

We describe efforts to improve the follow-up care of patients with abnormal Papanicolaou test results at an academic family practice center. Educational programs, formalized approaches to care, transportation assistance, and reminder systems can improve the outcome of programs screening for cervical cancer. ${ }^{1-11}$ Each of these techniques was used at the Shadyside Hospital Family Health Center to improve documentation of follow-up plans and to increase rates of return for repeat $\mathrm{Pa}$ panicolaou testing and colposcopy.

Follow-up of abnormal findings on Papanicolaou tests is obviously important. Women with atypia and dysplasia on Papanicolaou smears are more likely than women with normal smear findings to have frank carcinoma or to develop carcinoma. Fortunately, early discovery and treatment of cervical cancer produces very high cure rates. Furthermore, cryotherapy, loop excision, coniza-

Submitted, revised, 21 May 1997.

From the Primary Care Institute, Shadyside Hospital, Pittsburgh. Address reprint requests to Bruce Block, MD, 5215 Centre Ave, Pittsburgh, PA 15232. tion, and laser vaporization surgery can remove precancerous lesions and prevent development of carcinoma altogether in patients with abnormal cytologic findings on cervical biopsy.

Providing follow-up care for women with abnormal Papanicolaou test results can be challenging, however. Patients who have invasive cervical cancer often have a history of inadequate follow-up for abnormal findings on Papanicolaou smears. ${ }^{12,13}$ Poor rates of return for repeated Papanicolaou testing and for colposcopy have afflicted programs in diverse geographic and cultural settings. $8,11,14-17$ Obstacles to follow-up include conflicting health beliefs, cultural taboos, perceived interference with sexual relationships, denial driven by fear of cancer, inadequate transportation, forbidding costs, and inadequate guidance from health care providers. ${ }^{17-19}$

When it became apparent that patients at the Shadyside Hospital Family Health Center who had abnormal Papanicolaou test results were not receiving appropriate follow-up care, an iterative series of investigations and interventions were instituted in response. We report here those results. 
Background

Setting

The Family Health Center is staffed by more than 30 faculty and residents of the Family Practice Residency Program. Two obstetrician-gynecologists also work part-time at the center. Each year more than 7000 patients make more than 20,000 visits. Approximately 40 percent of these visits involve women older than 14 years. About 60 percent of our patients are indigent, and 50 percent of the adult women are African-American.

\section{Preliminary Investigation}

The center has used an automated medical record system $^{20}$ since 1985 , which includes a health surveillance tracking module to remind patients about overdue Papanicolaou testing. The routine screening protocol for cervical cancer stipulated a yearly Papanicolaou smear unless modified by the physician based on patient risk. In 1988, a telephone survey of 172 randomly selected women in the practice showed that 93 percent had received a screening Papanicolaou test in the previous 3 years.

In 1990, however, a review of the 197 women who had had abnormal test results between 1985 and 1990 showed that 70 patients (36 percent) were overdue for follow-up Papanicolaou testing or colposcopy. Patients who did not have abnormal cervical findings entered on the problem list were more likely to be overdue for follow-up (41 percent) than were patients who had problem list entries ( 25 percent). Follow-up plans, when documented at all, were often idiosyncratic and haphazard. Several charts contained notations of abnormal Papanicolaou smear results, initialed by the physician, without any evidence that the patient had been notified of the result.

\section{Initial Interventions and Results}

Concern about these findings led to educational sessions for physicians that stressed reasons for follow-up of mildly abnormal findings on Papanicolaou tests. A review of the literature and discussion with consultants provided the basis for a formalized approach to Papanicolaou test abnormalities. Physicians were prompted, in writing, about appropriate follow-up when they were given the test result (Figure 1). By the end of 1992, however, a review of 22 subsequent patients who had abnormal test results showed that there was proper documentation in only 15 cases ( 68 percent) and a combined colposcopy and Papanicolaou test follow-up success rate of 54 percent. For this reason, nursing and administrative staff developed a log book to track patient progress through the care system.

In March of 1994, immediately before the study interventions, we reviewed the status of 61 patients with abnormal Papanicolaou test findings dating back to August 1993. Documentation and notification of patients had improved to 78 percent and 71 percent, respectively. The colposcopy completion rate was 64 percent ( 29 of 45 patients), while the Papanicolaou test completion rate was 31 percent ( 5 of 16 ), a 56 percent combined follow-up success rate.

Based on this experience, the faculty and staff of the Family Health Center developed a more tenacious follow-up system using the automated tracking techniques and personalized reminders for patients and physicians. Colposcopy was added as an initial option for patients with atypical cells on $\mathrm{Pa}$ panicolaou smears because of concerns about the poor sensitivity of Papanicolaou screening.

\section{Methods}

All patients who had an abnormal Papanicolaou test result at the Family Health Center between 1 April 1994 and 31 March 1996 were entered into the case management system. Abnormal findings included all results described as atypical, dysplastic, atypical squamous cells of uncertain importance, low-grade squamous intraepithelial lesions, or high-grade squamous intraepithelial lesions showing koilocytosis, changes typical of human papillomavirus, or showing carcinoma in situ or higher grade carcinomas. Metaplasia and inflammation were not considered abnormal for the purpose of this study. Only patients of family physicians (faculty and residents) were included in the study because many patients seen by the part-time gynecologists were referred from other centers that did not use our case management system. $\mathrm{Pa}$ tient follow-up outcomes were assessed from chart review as of 31 March 1996.

\section{Care Intervention}

Automated exception reports were designed to list patients whose care was delinquent for critical steps in the case management process (Figure 2). The new case management system assured that 
TO: Residency Physicians

FROM: Ruth Branham - Quality Assurance \& Research Coordinator

RE: Abnormal Papanicolaou Test Follow-up

Your patient's cytology report shows an abnormal Papanicolaou test result. These are our recommended follow-up plans:

Atypia - atrophic

Estrogen \& Papanicolaou test in 6 weeks or colposcopy within 2 months

Atypia - inflammatory

Antibiotic \& Papanicolaou test in 6 weeks or colposcopy within 2 months

Repeated atypia

Colposcopy within 2 months

Koilocytosis, condyloma,

Colposcopy within 2 months or human papillomavirus

Mild dysplasia

Colposcopy within 2 months

Moderate to severe dysplasia

Colposcopy within 1 month

Carcinoma or cancer

Colposcopy within 2 weeks

You are responsible for contacting the patient about follow-up of her abnormal Papanicolaou test result. Please call the patient and explain your findings. Ask the patient to schedule the follow-up that you feel is appropriate. Fill out the information below for Ruth, telling her the patient's name and the follow-up plan you are recommending.

Ruth will check to see that the patient has scheduled an appointment and that it is for the appropriate time and place. She will also assure that a copy of the letter on the back of this memo will be sent to all patients for whom colposcopy is advised.

PLEASE FILL OUT THE INFORMATION BELOW AND RETURN TO RUTH:

Doctor's Name:

Patient's Name:

Date Patient Contacted:

Follow-up Plan Desired:

Comments:

Figure 1. Memorandum attached to abnormal Papanicolaou test result provided to physicians.

every patient with an abnormal Papanicolaou finding had a documented and verifiable followup plan developed by the patient's regular physician based on practice care standards. Patients without a plan appeared on an exception report (step R1, Figure 2). Physicians who were responsible for this lapse in planning received personal follow-up by the quality assurance coordinator, with backup from the medical director.

Patients with atypia who were scheduled to have follow-up Papanicolaou testing were entered into the Papanicolaou test-tracking system. If the patient did not receive a Papanicolaou test within the scheduled interval, an exception report (step
R2, Figure 2) brought the patient's status to the attention of the physician for personal action; the clerical assistant also generated a reminder letter for the patient.

Patients who were assigned to follow-up colposcopy received explanatory letters. Exception reports highlighted patients who failed to appear for colposcopy (step R3, Figure 2) or for discussion of biopsy results (step R4, Figure 2). These patients received reminder letters as well as telephone calls from office staff who were knowledgeable about colposcopy. Receptionists offered taxicab passes and expedited appointment times to overcome logistic obstacles. Patients who were 


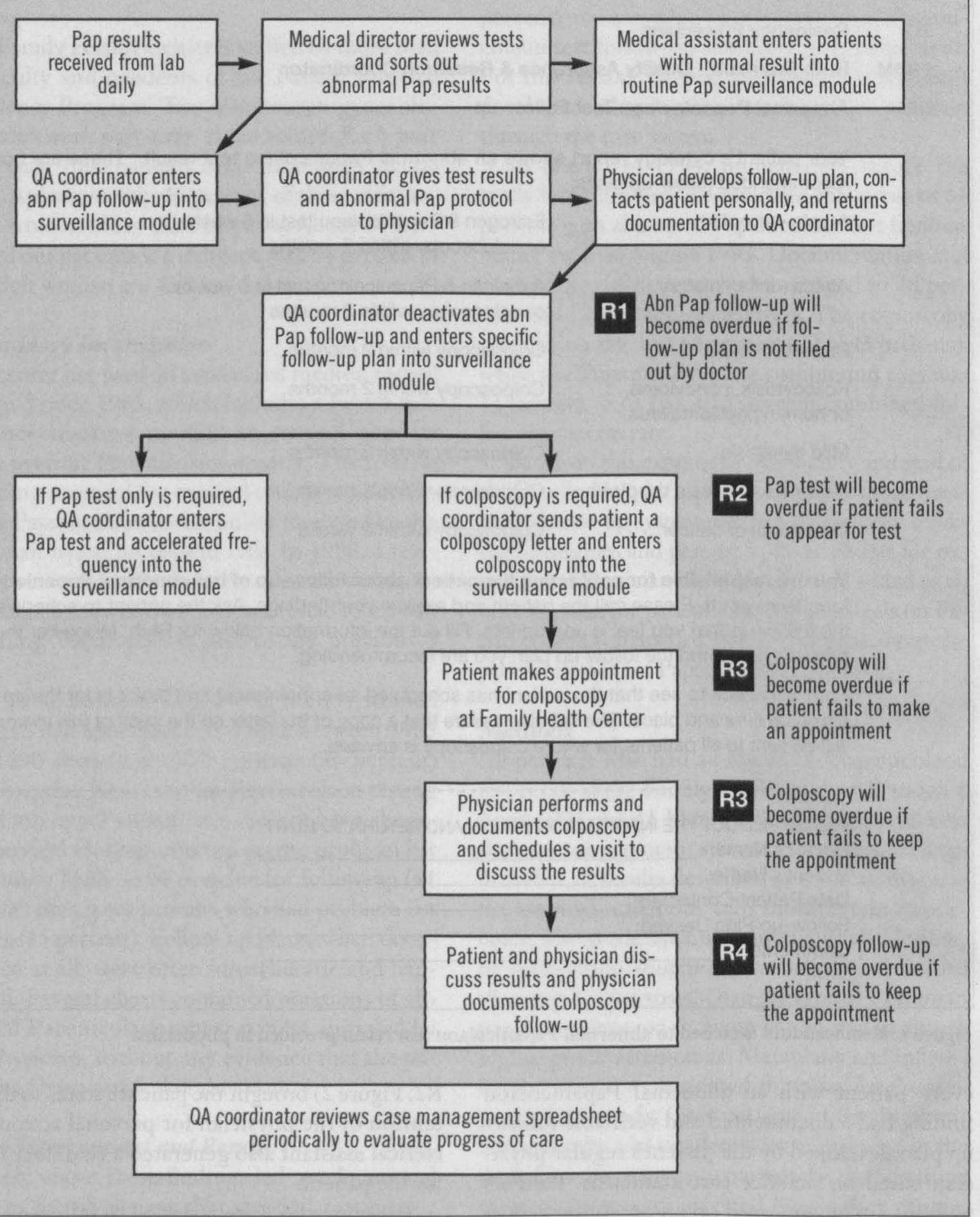

Figure 2. Case management system used in study. Pap - Papanicolaou, QA - quality assessment, abn - abnormal.

still reluctant to make appointments were referred to their own physicians at the center for further telephone discussion. Letters were sent to patients describing test results if no face-to-face discussion could be arranged. A spreadsheet provided a summary of case management efforts.

A tapered cell-sampling brush and a long- nosed spatula were used to collect cervical samples, except during pregnancy, when a swab was used instead of the brush. All smears were read by a single commercial laboratory or the hospital laboratory. The patient's family physician and a faculty gynecologist performed most colposcopies jointly in the Family Health Center or, rarely, in a 


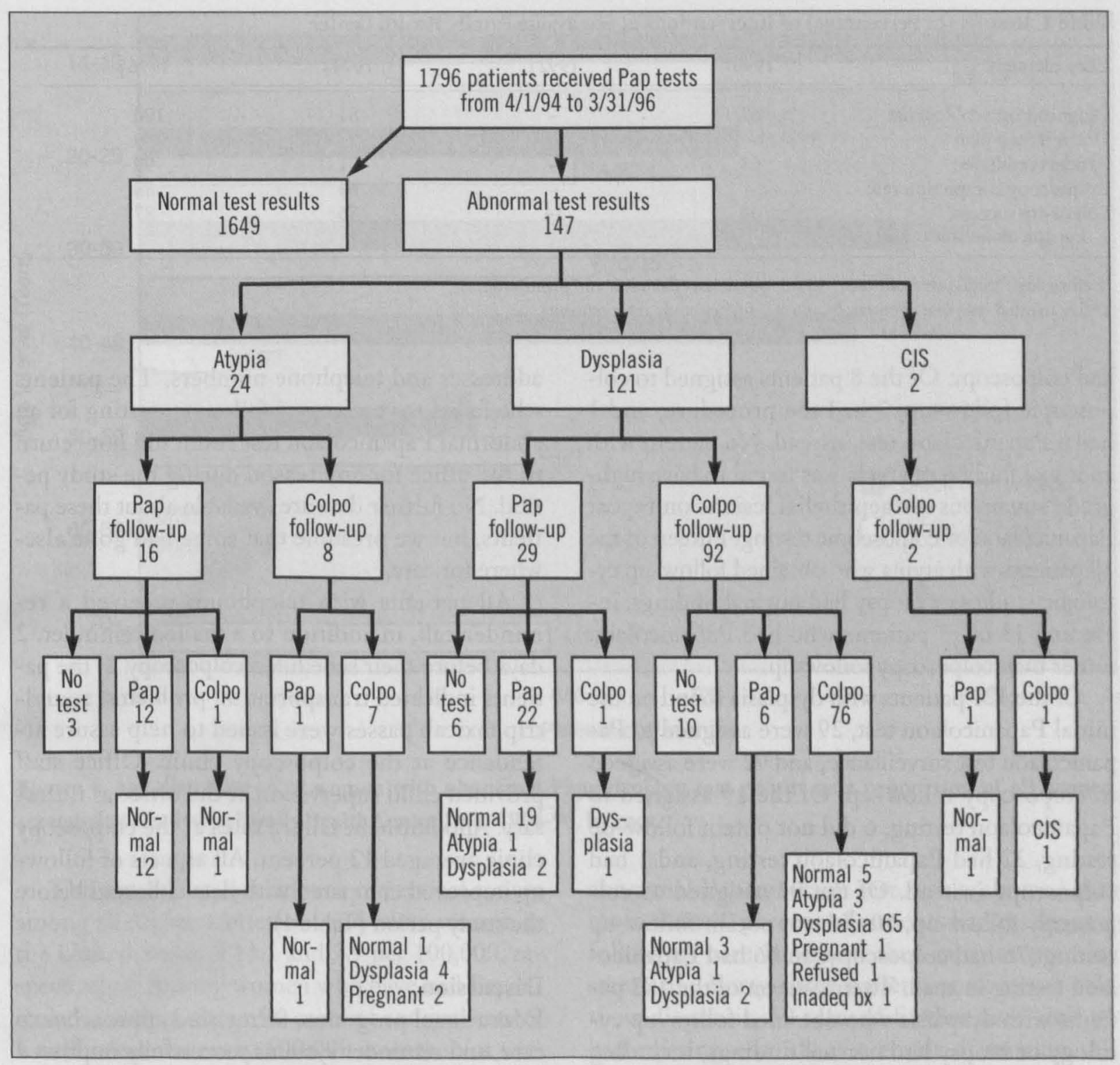

Figure 3. Summary of study results. Pap - Papanicolaou, CIS - carcinoma in situ, colpo - colposcopy, bx - biopsy.

hospital-based time-shared office when patient schedules required evening care. The hospital Pathology Department read all biopsies.

\section{Results}

Between 1 April 1994 and 31 March 1996, 1796 patients of family physicians received Papanicolaou tests at the Family Health Center. The average age of these patients was 33.2 years and 69 percent of patients were African-American. Yearly demographic studies of the center population confirm stability of race and age proportions since 1986.

Eight percent (147) of these patients had abnormal Papanicolaou test findings; 24 patients (16 percent) had atypia, 121 (82 percent) had dyspla- sia, and 2 (1 percent) had carcinoma in situ. All but 1 of these patients had a documented follow-up plan in the chart and in the automated tracking system (Figure 3).

Of the 45 patients who were assigned by their physician to Papanicolaou test surveillance, $36 \mathrm{pa}-$ tients completed at least one follow-up test, and 2 went on to colposcopy based on their follow-up results. Of 102 patients assigned to colposcopy, 85 had the procedure, 7 had Papanicolaou testing instead, and 10 were overdue for follow-up.

Of the 24 patients with atypia, 16 were assigned to follow-up Papanicolaou testing and 8 were assigned to colposcopic follow-up. Of the 16 patients assigned to Papanicolaou testing, 3 did not show up for the test, 12 had a Papanicolaou test, and 1 
Table 1. Results (in Percentage) of Interventions at Shadyside Family Health Center.

\begin{tabular}{|c|c|c|c|c|}
\hline Care Element & $1990^{*}$ & $1992 \dagger$ & $1994 \neq$ & $1996 \$$ \\
\hline Diagnosis on problem list & 67 & - & 81 & 100 \\
\hline Documented plan & - & 68 & 78 & 99 \\
\hline Patients reminded & 67 & - & 71 & 99 \\
\hline Colposcopy completion rate & - & - & 64 & 88 \\
\hline $\begin{array}{l}\text { Follow-up success, } \\
\text { Papanicolaou smear and c }\end{array}$ & 64 & 54 & 56 & 87 \\
\hline
\end{tabular}

*Before any formal interventions. †After physician education and prompting.

¥After manual case management by nurse. \$After study interventions.

had colposcopy. Of the 8 patients assigned to colposcopic follow-up, 7 had the procedure, and 1 had a Papanicolaou test instead. No patient with an initial finding of atypia was found to have highgrade squamous intraepithelial lesions on repeat Papanicolaou or colposcopic testing. Fifteen of the 19 patients with atypia who obtained follow-up cytologic studies or biopsy had normal findings, including 13 of 13 patients who had Papanicolaou rather than colposcopy follow-up.

Of the 121 patients with dysplasia found on the initial Papanicolaou test, 29 were assigned to $\mathrm{Pa}$ panicolaou test surveillance, and 92 were assigned to colposcopy follow-up. Of the 29 assigned to Papanicolaou testing, 6 did not obtain follow-up testing, 22 had Papanicolaou testing, and 1 had colposcopy instead. Of the 92 assigned to colposcopy follow-up, 10 did not obtain follow-up testing, 76 had colposcopy, and 6 had Papanicolaou testing instead. Twenty-seven of the $102 \mathrm{pa}-$ tients with dysplasia who obtained follow-up cytology or biopsy had normal findings, including 22 of 28 patients who had Papanicolaou rather than colposcopy follow-up.

Of the 2 patients with carcinoma in situ, both were assigned to colposcopy; 1 had Papanicolaou test follow-up and 1 had colposcopy.

All scheduled patients received automated reminders before the scheduled visits and telephone calls (or letters, if unreachable by telephone) after missed or canceled appointments. At all visits the physician received an automated reminder about the patient's status to take advantage of any opportunity to reinvolve the patient who was overdue for a follow-up Papanicolaou test. Only 2 patients came in for visits during the study period without receiving or being scheduled for follow-up testing.

All patients who were overdue for follow-up tests received telephone calls and reminder letters, although some had incorrect or nonfunctioning addresses and telephone numbers. The patients who failed to receive any follow-up testing for an abnormal Papanicolaou test result did not return to the office for any reason during the study period. No further data are available about these patients, but we presume that some had gone elsewhere for care.

All patients with telephones received a reminder call, in addition to a mailed reminder, 2 days before their scheduled colposcopy. If the patients indicated transportation problems, roundtrip taxicab passes were issued to help assure attendance at the colposcopy clinic. Office staff provided child supervision at the office as necessary. Appointment failure rates at the colposcopy clinic averaged 12 percent. All aspects of followup improved compared with data collected before the study period (Table 1).

\section{Discussion}

Educational programs, formalized approaches to care, and reminder systems successfully improved the outcomes of the cervical cancer screening program at the Family Health Center. The success of cervical cancer prevention programs depends on the breadth of Papanicolaou test screening, the completeness of follow-up of abnormal findings, the accuracy of the follow-up tests, and ultimately the effectiveness of therapeutic interventions.

\section{Breadth of Papanicolaou Test Screening}

Incomplete Papanicolaou testing of community populations occurs most commonly among African-Americans, elderly patients, and patients living in rural areas. ${ }^{21}$ Although the service population of Shadyside Family Health Center is urban and mainly African-American, a study done in 1988 at the center showed that 93 percent of eligible patients had Papanicolaou test screening in the preceding 3 years. 


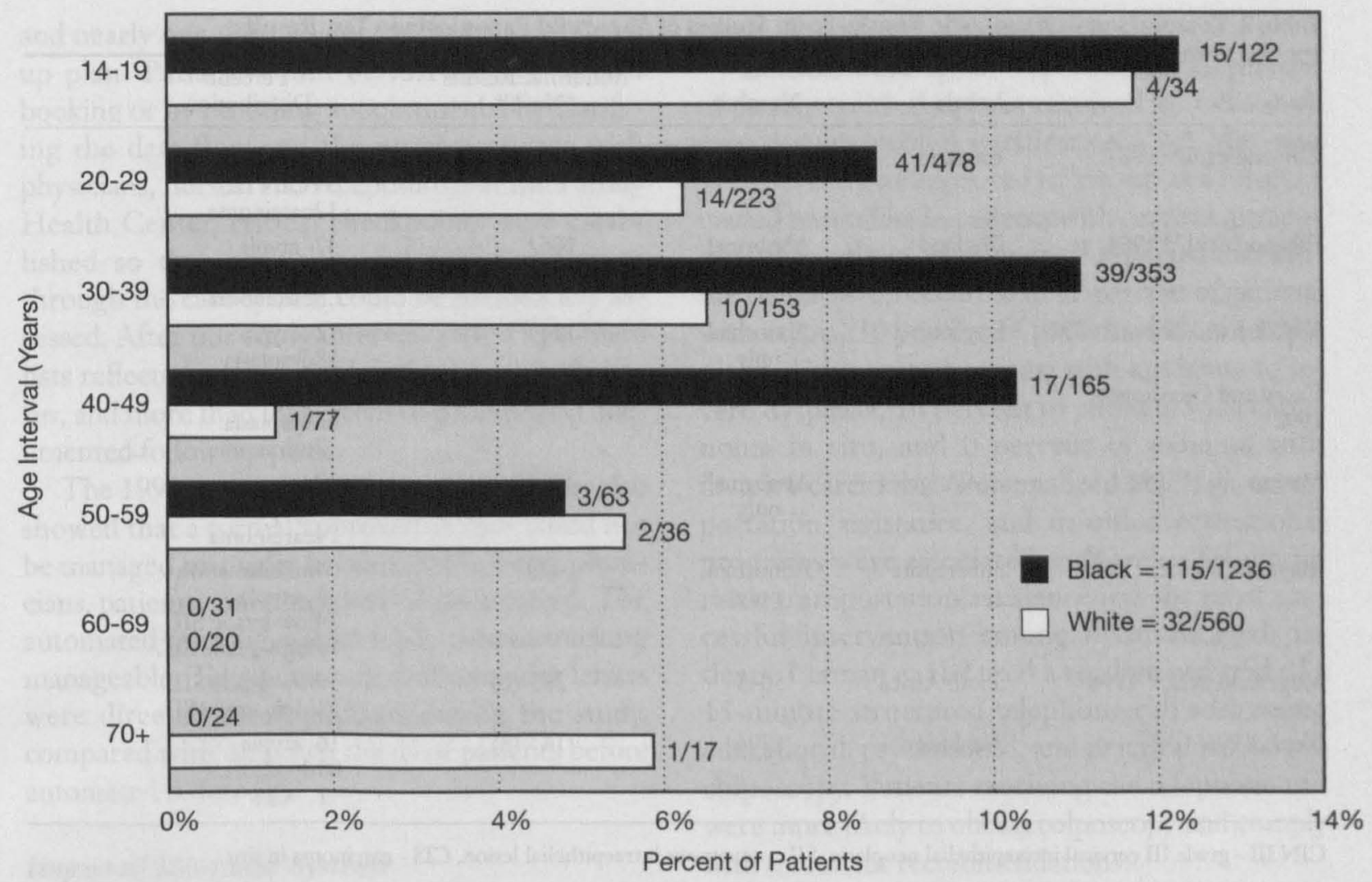

Figure 4. Age distribution of women with abnormal Papanicolaou test results as a proportion of all women screened at Shadyside Family Health Center from 1994-96, by race.

The age-adjusted incidence of cervical cancer among all African-American and white women in the United States is 13.1 and 7.9 per 100,000 , respectively.22 Among women who have had Papanicolaou testing, about 96 cases of cervical cancer would occur for every 100,000 smears. Not surprisingly, we found no cases of cancer among the 1797 women who received Papanicolaou tests.

African-American and young patients at the Family Health Center had higher rates of abnormal findings on Papanicolaou tests than other patients (Figure 4). Studies of the results of Papanicolaou test screening done elsewhere reveal similar rates of abnormal findings, but frequencies of cytologic diagnoses varied widely, probably reflecting differences in classification rather than biologic divergence (Table 2).

\section{Problems With Follow-up Testing}

Failure to notify patients, failure to make and keep appointments, and failure to obtain the proper follow-up test plague all cervical-cancer-screening programs. Ellwood et al, ${ }^{14}$ in a prospective study of 1062 patients with 2 years elapsed since the first abnormal Papanicolaou test result, showed that more than 40 percent of patients had inadequate follow-up care based on then-current practice standards. Forty-three patients were never told their diagnosis, 30 refused further care, and 86 had missing records as a result of moving. All these patients had a regular source of medical care available at nominal cost. In Australia Hunt et al ${ }^{15}$ found that 22 (18 percent) of 121 women with atypia and 8 ( 8 percent) of 100 women with grade 1 cervical intraepithelial neoplasia on their Papanicolaou test had no follow-up testing within the 12 months after their original test.

Schofield et al, ${ }^{16}$ who interviewed 315 women in Australia who had abnormal Papanicolaou test results, reported that 7 percent had not been notified at all and an additional 11 percent were unaware of abnormal results. Only 60 percent of patients received the follow-up care recommended by their physician. Marcus et al, ${ }^{8}$ who examined follow-up care for more than 2000 patients in 12 clinics in Los Angeles, found a 29 percent failure rate. Carey and Gjerdingen, ${ }^{17}$ in a study of 190 Minnesota women with abnormal Papanicolaou 
Table 2. Comparison of Diagnostic Results From Studies of Abnormal Papanicolaou Test Results.

\begin{tabular}{|c|c|c|c|c|}
\hline Study, Year & Locale & Number & $\begin{array}{c}\text { Abnormal Results } \\
\text { No. (\%) }\end{array}$ & $\begin{array}{c}\text { Percent/ } \\
\text { Distribution }\end{array}$ \\
\hline Ellwood et al, ${ }^{14} 1984$ & England & 4280 & $1774(41)$ & $\begin{array}{l}49 \text { atypia } \\
39 \text { CIN III } \\
12 \text { carcinoma }\end{array}$ \\
\hline Ellwood et al, ${ }^{14} 1984$ & England & $\begin{array}{l}\text { Abnormal } \\
\text { only }\end{array}$ & 1062 & $\begin{array}{l}47 \text { atypia } \\
47 \text { dysplasia } \\
2.5 \text { carcinoma }\end{array}$ \\
\hline Woolley and Talbot, ${ }^{5} 1990$ & England & $\begin{array}{l}\text { Abnormal } \\
\text { only }\end{array}$ & 1064 & $\begin{array}{l}72 \text { atypia } \\
18 \text { dysplasia }\end{array}$ \\
\hline $\begin{array}{l}\text { Carey and Gjerdingen, }{ }^{17} \\
1992\end{array}$ & Minnesota & 1794 & $190(11)$ & $\begin{array}{l}36 \text { atypia } \\
62 \text { dysplasia } \\
\text { 2carcinoma }\end{array}$ \\
\hline Marcus et al, ${ }^{8} 1992$ & Los Angeles & $\begin{array}{l}\text { Abnormal } \\
\text { only }\end{array}$ & 2044 & $\begin{array}{l}39 \text { atypia } \\
57 \text { dysplasia } \\
\text { 4carcinoma }\end{array}$ \\
\hline Mayeaux Jr et al, ${ }^{25} 1995$ & Shreveport & $\begin{array}{l}\text { Abnormal } \\
\text { only }\end{array}$ & 428 & $\begin{array}{l}\text { 4inflammation } \\
4 \text { atypia } \\
\text { 74low-grade SIL } \\
\text { 18high-grade SIL }\end{array}$ \\
\hline Megevand et al, ${ }^{11} 1996$ & South Africa & 5045 & $293(6)$ & $\begin{array}{l}\text { 59low-grade SIL } \\
\text { 41high-grade SIL }\end{array}$ \\
\hline Block, 1996 & Pittsburgh & 1796 & $147(8)$ & $\begin{array}{l}16 \text { atypia } \\
83 \text { dysplasia } \\
1 \text { CIS }\end{array}$ \\
\hline
\end{tabular}

CIN III - grade III cervical intraepithelial neoplasia, SIL - squamous intraepithelial lesion, CIS - carcinoma in situ.

test results, found a 15 percent follow-up failure rate. Megevand et al ${ }^{11}$ documented a 66 percent follow-up failure rate among patients in South Africa with high-grade squamous intraepithelial lesions on Papanicolaou testing. The follow-up failure rate after multifaceted intervention in our study ( 12 percent) is better than those previously reported.

\section{Impact of Educational Programs}

The most effective educational interventions for clinicians provide definitive information in the midst of decision-making. ${ }^{23}$ The clinical decisionsupport system for this study was the sheet attached to the Papanicolaou test result that told physicians what to do for each cytologic finding (Figure 1). Recommendations for follow-up of atypia encouraged the clinician to obtain a repeated Papanicolaou test after therapeutic intervention or to proceed directly to colposcopy. $\mathrm{Pa}$ tients with higher grade abnormalities were to have colposcopy within the intervals corresponding to the grade of the lesions found. For patients with dysplasia, physicians chose follow-up plans that were consistent with the program recommendations in 92 of 121 (76 percent) cases. Discrepant plans occurred only in patients with mild dysplasia who were assigned to Papanicolaou test rather than colposcopy, a recommendation that was still under debate in the literature at the time.

Educational options for patients in our program included discussion with their physician, discussion with an office nurse or a specially trained medical assistant, and an explanatory letter. Paskett et al ${ }^{l}$ improved follow-up Papanicolaou test rates for women with abnormal cytologic findings on cervical smears by 13 percent with a pamphlet and reminder sticker. Stewart et $\mathrm{al}^{2}$ documented a 30 percent higher follow-up rate in women who were given explanatory brochures when they booked their colposcopy appointment. We did not study educational input to patients as a separate factor influencing follow-up rate.

\section{Impact of Formalized Care Management}

In complex and distracting practice environments, an intention to treat is difficult to implement without an organized and visible approach to care. Before formalizing care in our center, physicians could respond inadequately to abnormal Papanicolaou test findings without discovery or correction of the error: one third of patients with abnormal Papanicolaou smear findings did not have it so designated on the problem lists in their charts, 
and nearly one third had no documented followup plan. Patients could be lost to care by not booking or by canceling appointments. By clarifying the data flow and the process of care with physicians, nurses, and receptionists at the Family Health Center, critical checkpoints were established so that physician and patient progress through the care system could be periodically assessed. After our study interventions all problem lists reflected a patient's abnormal test result status, and more than 99 percent of patients had documented follow-up plans.

The 1992 reevaluation of care at the center also showed that a formal approach to care could not be managed manually because of the many physicians, patients, and procedural steps involved. The automated tracking system made patient tracking manageable. Telephone calls and reminder letters were directed to all patients during the study, compared with only two thirds of patients before automated follow-up.

\section{Impact of Reminder Systems}

Prompting techniques provide a critical form of logistic support to well-intentioned care. Reminders can improve Papanicolaou screening, encourage follow-up of abnormal test results, and ensure colposcopic screening, but only when community access to care can be assured. Michielutte et $\mathrm{al}^{3}$ determined that 80 percent of patients with grade III or greater cervical intraepithelial neoplasia responded to mailed or telephone reminders for further examination. Ten percent of patients had no follow-up documented in their charts and could not be reached for the study. Frisch ${ }^{4}$ used a care protocol that included letters and telephone calls and reduced follow-up failures from 31 percent to 11 percent.

Woolley and Talbot ${ }^{5}$ described failure of follow-up in 55 percent of patients with atypia and 20 percent of patients with moderate dysplasia, but only 3 percent of patients with severe dysplasia for whom an intensive case tracking system was used failed to return for follow-up. Mitchell and Medley ${ }^{6}$ sent reminders to the patient's practitioner if the patient was more than 3 months overdue for follow-up of the abnormal Papanicolaou test findings. Patients with atypia and mild dysplasia had a failure-to-return rate of 18 percent, and those with moderate dysplasia, 10 percent. Letters to the physician decreased the failure rate by about 20 percent.

Laedtke and Dignan 7 reported that 22 percent of patients with dysplasia received no follow-up care despite mailed notification. Del Mar and Wright ${ }^{9}$ showed improved follow-up as a result of mailed reminders in patients with cervical intraepithelial neoplasia. Marcus et $\mathrm{al}^{8}$ reported that failure of follow-up occurred in 32 percent of patients with atypia, 30 percent of patients with mild dysplasia, 22 percent of patients with moderate to severe dysplasia, 16 percent of patients with carcinoma in situ, and 0 percent of patients with invasive carcinoma. Personalized mailings, transportation assistance, and in-office educational programs were associated with better follow-up rates; transportation assistance was the most successful intervention among disadvantaged patients. Lerman et a ${ }^{10}$ used a randomized trial of a 15-minute structured telephone call addressing educational, psychosocial, and practical barriers to colposcopy. Patients receiving the telephone call were more likely to obtain colposcopy and comply with treatment recommendations.

Overall follow-up success rates and colposcopy completion rates increased dramatically in our study with the use of education, transportation assistance, and reminders. Although 36 percent of patients with abnormal smear findings had been overdue for follow-up in 1990 , only 13 percent were overdue after our interventions in $1996 . \mathrm{Pa}$ tients assigned to Papanicolaou testing for followup of abnormal smear findings failed to receive a test in 9 of 45 (20 percent) cases, but those assigned to colposcopy follow-up failed to receive a test in only 10 of 102 (10 percent) of cases. Appointment failure rates at the colposcopy clinic dropped from 56 percent in 1993 to 12 percent in 1996.

As these data show, patients with the most worrisome screening Papanicolaou test results had the most successful follow-up rates; presumably the extra effort of medical offices and the extra concern of affected patients conspired to produce this effect. Conversely, insufficient concern on the part of physicians and patients could contribute to the inadequate follow-up of atypical Papanicolaou smears shown in other studies. Sequential Papanicolaou test follow-up of abnormal Papanicolaou smear findings is harder to implement than is colposcopy in practices that do their colposcopy on site. 
Accuracy of Follow-up Testing

Unfortunately, the cytologic classification of Papanicolaou test results does not predict with precision the subsequent result of cervical biopsy. Slawson et $\mathrm{a}^{24}$ showed that a single follow-up $\mathrm{Pa}$ panicolaou smear failed 50 percent of the time to detect biopsy-proved cervical intraepithelial neoplasia in patients with atypical squamous cells on initial Papanicolaou testing. Mayeaux et al ${ }^{25}$ performed simultaneous Papanicolaou testing and colposcopic biopsy on 428 patients with initially abnormal cervical smear findings, including inflammation, atypia, and low- and high-grade squamous intraepithelial lesions. He found in patients with biopsy-proven high-grade squamous intraepithelial lesions that follow-up Papanicolaou smears rather than colposcopic biopsy would have mistakenly underestimated the severity of 87 percent of the lesions and would have missed abnormalities altogether in 23 percent.

Follow-up Papanicolaou testing of patients with abnormal smear findings detected very little disease in our study when compared with colposcopic biopsy. Of 42 patients who had follow-up Papanicolaou testing, 86 percent had normal findings on cytology studies, but only 9 percent ( 7 of 83) of colposcopy patients had normal biopsy findings. This discrepancy was just as remarkable for patients who initially had atypia (100 percent of the Papanicolaou findings were normal, but only 33 percent of the biopsy results were normal) as it was for those who initially had dysplasia (79 percent normal Papanicolaou findings, but 7 percent normal biopsy findings).

Multiple sequential Papanicolaou tests should be more sensitive than single tests in the follow-up of initially abnormal smear findings, but they are logistically even more difficult to complete. For practices that offer colposcopy on site, colposcopic biopsy is a better follow-up choice than repeated Papanicolaou testing, even for so-called lower grade abnormalities. Among patients with highgrade squamous intraepithelial lesions, Megevand et al $^{11}$ were able to reduce a follow-up failure rate from 66 percent to 50 percent with intensive public health intervention but reduced the rate to 3 percent using a mobile van with same-day Papanicolaou testing, colposcopy, and treatment.

\section{Sources of Error}

The findings we describe are observations of a busy and sometimes chaotic practice. Information collected before the formal interventions was generated from quality-improvement data, which lack the rigor of formal research. Although using quality-improvement data might enhance the external validity of the results for urban family practice centers, it does limit the reliability of pre- and post-intervention comparisons. New interventions are implemented in a practice setting only when existing systems are functioning poorly; apparent improvements can be related to changes in effort, service demand, and staffing rather than the studied interventions.

Presumably the outcome of follow-up efforts improves with time for patients who remain accessible to the practice. The follow-up interval in this 2-year study varied from 1 to 24 months for individual patients; thus the follow-up outcomes reported might underrepresent the benefit of persistent efforts to involve patients in care. Some patients lost to follow-up could have obtained appropriate care elsewhere as a result of interventions initiated by our practice, leading to an underestimation of the success of interventions.

\section{Conclusion}

The combination of a well-functioning community Papanicolaou screening program, well-educated clinicians, well-organized care processes, and well-designed tracking and reminder systems can reduce follow-up failure rates dramatically. In low-income communities such as ours, reduction of financial barriers, transportation assistance, and child care further improve the chances of effective follow-up. Colposcopic biopsy appears to be more accurate than sequential Papanicolaou smears in detecting treatable precursors of cervical cancer, and doing a colposcopic biopsy is logistically simpler in practices that perform colposcopy on site.

Eric Miller, MD, provided important assistance in data analysis and review.

\section{References}

1. Paskett ED, White E, Carter WB, Chu J. Improving follow-up after an abnormal Papanicolaou smear: a randomized controlled trial. Prev Med 1990;19:63041.

2. Stewart DE, Buchegger PM, Lickrish GM, Sierra S. The effect of educational brochures on follow-up compliance in women with abnormal Papanicolaou smears. Obstet Gynecol 1994;83:583-5. 
3. Michielutte R, Diseker R, Young LD, May WJ. Noncompliance in screening follow-up among family planning clinic patients with cervical dysplasia. Prev Med 1985;14:248-58.

4. Frisch LE. Effectiveness of a case management protocol in improving follow-up and referral of Papanicolaou smears indicating cervical intraepithelial neoplasia. J Am Coll Health 1986;35:112-5.

5. Woolley PD, Talbot MD. Experience in Sheffield: follow-up of abnormal cervical cytology. Int J STD AIDS 1990;1:95-7.

6. Mitchell H, Medley G. Adherence to recommendations for early repeat cervical smear tests. BMJ 1989;298:1605-7.

7. Laedtke TW, Dignan M. Compliance with therapy for cervical dysplasia among women of low socioeconomic status. South Med J 1992;85:5-8.

8. Marcus AC, Crane LA, Kaplan CP, Reading AE, Savage E, Gunnning J, et al. Improving adherence to screening follow-up among women with abnormal Papanicolaou smears: results from a large clinicbased trial of three intervention strategies. Med Care 1992;30:216-30.

9. Del Mar CB, Wright RG. Notifying women of the results of their cervical smear tests by mail: does it result in decreased loss to follow-up of abnormal smears? Aust J Public Health 1995;19:211-3.

10. Lerman C, Hanjani P, Caputo C, Miller S, Delmoor $E$, Nolte $S$, et al. Telephone counseling improves adherence to colposcopy among lower-income minority women. J Clin Oncol 1992;10:330-3.

11. Megevand E, Van Wyk W, Knight B, Bloch B. Can cervical cancer be prevented by a see, screen, and treat program? A pilot study. Am J Obstet Gynecol 1996;174:923-8.

12. Brown RK, Barker WH Jr. Pap smear screening and invasive cervical cancer. J Fam Pract. 1982;15:875-9.

13. Carmichael JA, Jeffrey JF, Steele HD, Ohlke ID. The cytologic history of 245 patients developing invasive cervical cancer. Am J Obstet Gynecol 1984;148:685-90.

14. Ellwood JM, Cotton RE, Johnson J, Jones GM, Curnow J, Beaver MW. Are patients with abnormal cervical smears adequately managed? BMJ 1984;
229:891-4.

15. Hunt JM, Irwig LM, Towler BP. The management of women with initial minor Pap smear abnormalities. Med J Aust 1994;160:558-63.

16. Schofield MJ, Sanson-Fisher R, Halpin S, Redman $S$. Notification and follow-up of Pap test results: current practice and women's preferences. Prev Med 1994;23:276-83.

17. Carey P, Gjerdingen DK. Follow-up of abnormal Papanicolaou smears in women of different races. J Fam Pract 1993;37:583-7.

18. Kavanagh AM, Simpson JM. Predicting nonattendance for colposcopy clinic follow-up after referral for an abnormal Pap smear. Aust N Z J Public Health 1996;20:266-71.

19. Bennetts A, Irwig L, Oldenburg B, Simpson JM, Mock P, Boyes A, et al. PEAPS-Q: a questionnaire to measure the psychosocial effects of having an abnormal Pap smear. Psychosocial Effects of Abnormal Pap Smears Questionnaire. J Clin Epidemiol 1995;48:1235-43.

20. Block B, Brennan JA. Automated health surveillance. Fam Med 1988;20:377-80.

21. US Preventive Services Task Force. Guide to clinical preventive services: report of the US Preventive Services Task Force. 2nd ed. Baltimore: Williams \& Wilkins, 1996:105-17.

22. Martin LM, Parker SL, Wingo PA, Heath CW Jr. Cervical cancer incidence and screening: status report on women in the United States. Cancer Pract 1996;4:135-40.

23. McDonald CJ, Hui SL, Smith DM, Tierney WM, Cohen SJ, Weinberger $M$, et al. Reminders to physicians from an introspective computer medical record: a two-year randomized trial. Ann Intern Med 1984; 100:130-8.

24. Slawson DC, Bennett JH, Herman JM. Follow-up Papanicolaou smear for cervical atypia: are we missing significant disease? A HARNET Study. J Fam Pract 1993;36:289-93.

25. Mayeaux EJ Jr, Harper MB, Abreo F, Pope JB, Phillips GS. A comparison of the reliability of repeat cervical smears and colposcopy in patients with abnormal cervical cytology. J Fam Pract 1995;40:57-62. 